\title{
Determination of the diffusion constant using phase-sensitive measurements
}

\author{
I. M. Vellekoop, P. Lodahl, and A. Lagendijk \\ Complex Photonic Systems, Faculty of Science and Technology, University of Twente, P.O. Box 217, 7500 AE Enschede, The Netherlands
}

(Received 8 October 2004; published 12 May 2005)

\begin{abstract}
We apply a pulsed-light interferometer to measure both the intensity and the phase of light that is transmitted through a strongly scattering disordered material. From a single set of measurements we obtain the timeresolved intensity, frequency correlations and statistical phase information simultaneously. We compare several independent techniques of measuring the diffusion constant for diffuse propagation of light. By comparing these independent measurements, we obtain experimental proof of the consistency of the diffusion model and corroborate phase statistics theory.
\end{abstract}

DOI: 10.1103/PhysRevE.71.056604

PACS number(s): 42.25.Dd, 42.30.Ms, 61.43.Gt

\section{INTRODUCTION}

Diffusion is one of the most widely encountered phenomena in physics. The dissolving of sugar in water, the transfer of heat in a wire and the transport of carriers in a photodiode are all examples of diffusion. These processes are all described by the same diffusion equation. This equation also describes the diffuse transport of waves in disordered scattering materials. An example of a diffusing wave is the transport of light through a cloud or a colloid suspension. Wave diffusion is not limited to light; acoustic waves, microwaves, quantum particles or even seismic waves behave completely analogously.

The last couple of decennia wave diffusion has been of strong interest both from applied as well as fundamental points of view. In contrast to classical particle diffusion, wave diffusion is influenced by interference. The recognition that phase plays an important role in wave diffusion forms the basis for applications like diffusing wave spectroscopy [1] and optical coherence tomography [2], which are invaluable tools in the analysis of colloidal systems and in the optical imaging of biological tissue. Fundamental interest is motivated especially by the parallels between light diffusion and transport of electrons in mesoscopic systems. These parallels have been demonstrated by the observation of the optical equivalents of universal conductance fluctuations [3] and weak localization $[4,5]$.

Multiply scattering media are characterized by the transport mean free path $l$ (the average distance a wave travels through the medium before becoming diffuse) and the diffusion constant $D$ (the rate at which diffuse waves spread over the medium). For electrons $l$ can be considerably smaller than the wavelength $\lambda$ of the electron. When $(l \lesssim \lambda / 2 \pi)$, electrons become localized and the diffusion constant vanishes [6,7]. This breakdown of diffusion is called Anderson localization. Anderson localization of microwaves has been observed in quasi-1D systems [8]. Observations at optical wavelengths [9], however, remain under debate [10].

In quasi-1D microwave experiments, localization was shown to have a distinct effect on the statistical distributions of the intensity [8] and the phase [11]. Recently it has become possible to perform dynamic electric field measurements also in the optical regime, which allow a study of the optical phase $[12,13]$. These types of measurements provide a direct measurement of the phase of diffusing waves and they can give unambiguous proof of the presence of Anderson localization of light.

Here we report our optical experiments that thoroughly test wave-diffusion theory by measuring the amplitude and the phase of light transmitted through a strongly scattering, nonlocalizing medium. Using the technique of ultrashort pulse interferometry [12], we have access to the timeresolved intensity, the frequency-resolved intensity and the statistical distribution of the phase delay time. We demonstrate five different ways of extracting the diffusion constant from this multitude of experimental data. By comparing the results of these five different methods, we test the diffusion model thoroughly and moreover show how to interpret timeresolved and frequency-resolved measurements consistently.

In Sec. II of this paper we present a model for diffusion through a slab. From this model we will derive both the frequency-dependent and the time-dependent behavior and identify characteristic parameters that can be extracted from experimental data. The setup for measuring both the amplitude and phase of transmitted light is described in Sec. III. In Sec. IV we present our results and devote special attention to the comparison of different techniques to measure the diffusion constant. Our conclusions are given in Sec. V.

\section{THEORY}

\section{A. An exact solution to the diffusion equation}

We consider the diffusion of scalar waves through a slab of random material. The slab fills the space $0 \leqslant z \leqslant L$ and is infinite in the other directions. In this geometry it is convenient to use Fourier transformed coordinates $\mathbf{q}_{\perp} \equiv\left(q_{x}, q_{y}\right)$ for the transverse directions. The slab is illuminated from the left $(z<0)$ by a pulse at time $t=0$. Since the incident light quickly loses its directionality due to scattering, it is possible to model the incoming light by a diffuse source inside the material. In this paper we assume isotropic scattering. The inclusion of anisotropic scattering in the source function and the description of anisotropic diffusion are tremendous, and basically unsolved complications. At this point we use a source located at a depth $z_{0} \approx l[14]$. Later we will use a more 
sophisticated source. Under these conditions, the ensemble averaged energy density of diffuse light $I$ is described by the diffusion equation [15],

$$
\left[\partial_{t}+D\left(\mathbf{q}_{\perp}^{2}+\alpha^{2}-\partial_{z}^{2}\right)\right] I\left(\mathbf{q}_{\perp}, z ; t\right)=\delta\left(z-z_{0}\right) \delta(t) S\left(\mathbf{q}_{\perp}\right) .
$$

In this equation $\alpha \equiv \sqrt{3 /\left(l l_{a}\right)}$ is the absorption coefficient corresponding to an absorption mean free path $l_{a}$. The right hand side of Eq. (1) is the source term, where $S\left(\mathbf{q}_{\perp}\right)$ describes the transverse distribution of the source and has the unit of energy. The total energy in the source pulse is given by $S\left(\mathbf{q}_{\perp}=0\right)$.

The propagation of light is affected by the boundaries of the slab. It has been shown $[16,17]$ that reflections at the surfaces impose mixed boundary conditions on the diffusion equation,

$$
\begin{gathered}
\partial_{z} I\left(\mathbf{q}_{\perp}, 0 ; t\right)=I\left(\mathbf{q}_{\perp}, 0 ; t\right) / z_{e 1}, \\
-\partial_{z} I\left(\mathbf{q}_{\perp}, L ; t\right)=I\left(\mathbf{q}_{\perp}, L ; t\right) / z_{e 2},
\end{gathered}
$$

where $z_{e 1}$ and $z_{e 2}$ are so called extrapolation lengths. In the diffusion model, their values are given by $z_{e 1,2}=2 l(1$ $\left.+R_{1,2}\right) / 3\left(1-R_{1,2}\right)$. The reflection coefficients $R_{1}$ and $R_{2}$ correspond to the left and the right boundaries, respectively. These coefficients can be estimated from Fresnel's law using the refractive indices of the dielectrics outside of the slab and the effective index of the random medium [17].

We solve the diffusion equation [Eq. (1)] with mixed boundary conditions analytically in the frequency domain. This solution can conveniently be used to find the field correlation function, the total transmission and the average diffuse traversal time. We use the same approach as in [17], with the exception that we extend the model to allow for different extrapolation lengths at the two boundaries and use an exponential distribution of the source intensity.

When Eq. (1) is Laplace transformed with respect to $t$, an expression for the energy density $\tilde{I}$ can be found directly [18],

$$
\tilde{I}(\eta, z)=\frac{S\left(\mathbf{q}_{\perp}\right)}{2 D \eta}\left[e^{-\eta\left|z-z_{0}\right|}+A(\eta) e^{\eta z}+B(\eta) e^{\eta(L-z)}\right],
$$

where we have defined $\eta \equiv \sqrt{i \Omega / D+\mathbf{q}_{\perp}^{2}+\alpha^{2}}$. The Laplace transform parameter $\Omega$ describes the frequency of intensity oscillations and is much smaller than the optical frequency of the field $\omega . A$ and $B$ are found from the boundary conditions (2a) and (2b) after tedious algebra,

$$
\begin{gathered}
A(\eta)=\frac{\gamma^{+}\left(z_{0}\right)-2\left[z_{e 1} \eta+1\right] e^{\eta z_{0}}}{\gamma^{-}(L) e^{\eta L}}, \\
B(\eta)=\frac{\gamma^{+}\left(L-z_{0}\right)-2\left[z_{e 2} \eta+1\right] e^{\eta\left(L-z_{0}\right)}}{\gamma^{-}(L) e^{\eta L}},
\end{gathered}
$$

where we defined function $\gamma^{ \pm}$as

$$
\gamma^{ \pm}(x) \equiv\left(z_{e 1} \eta+1\right)\left(z_{e 2} \eta+1\right) e^{\eta x} \pm\left(z_{e 1} \eta-1\right)\left(z_{e 2} \eta-1\right) e^{-\eta x} .
$$

We now have the exact solution to the diffusion equation with mixed boundary conditions. In order to find the trans- mitted intensity flux, we calculate the forward flux $\widetilde{J}_{z}$ $=-D \partial_{z} \tilde{I}$ at the slab surface $z=L$,

$$
\tilde{J}_{z}(\eta, L)=S\left(\mathbf{q}_{\perp}\right) \frac{F_{z_{0}}(\eta)-F_{z_{0}}(-\eta)}{\gamma^{-}(L)},
$$

where $F_{z_{0}}$ is given by

$$
F_{z_{0}}(\eta) \equiv\left[z_{e 1} \eta+1\right] e^{\eta z_{0}} .
$$

Equation (7) describes the transmission for a source located at depth $z_{0}$. A more realistic and more sophisticated model assumes an exponential distribution of the source light. The exponential distribution models how light becomes diffuse by being scattered out of the incoming coherent beam. We adapt Eq. (7) for the exponential source model by convolving $F_{z_{0}}$ with a (normalized) exponential source function,

$$
\begin{aligned}
F_{l}(\eta) & =\int_{0}^{L} d z_{0} \frac{\exp \left(-z_{0} / l\right)}{l[1-\exp (-L / l)]} F_{z_{0}}\left(\eta ; z_{0}\right) \\
& =\frac{1-\exp (L \eta-L / l)}{1-\exp (-L / l)} \frac{1+z_{e 1} \eta}{1-l \eta} .
\end{aligned}
$$

We obtain the transmitted flux for the exponential source from Eq. (7) by simply replacing $F_{z_{0}}$ by $F_{l}$.

An important quantity in the analysis of random media is the total transmission. The total transmission is found by integrating the flux over the whole back surface of the sample (this corresponds to taking $\mathbf{q}_{\perp}=0$ ) and integrating over time $(\Omega=0)$. The ensemble averaged total transmission coefficient $T_{\text {tot }}$ is therefore defined as

$$
T_{\text {tot }} \equiv \frac{\widetilde{J}_{z}\left(\mathbf{q}_{\perp}=0, \Omega=0\right)}{S\left(\mathbf{q}_{\perp}=0\right)}=\frac{\widetilde{J}_{z}(\eta=\alpha)}{S\left(\mathbf{q}_{\perp}=0\right)} .
$$

Neglecting absorption, the equation is evaluated to reproduce the well known result [19]

$$
T_{\text {tot }}=\frac{l+z_{e 1}}{L+z_{e 1}+z_{e 2}}+O(\exp (-L / l)) .
$$

This relation between $T_{\text {tot }}, l$ and $L$ is often used to determine the mean free path experimentally by varying $L$.

Next, we calculate the electric field correlation function $C_{E}$ for the transmitted light. This correlation function contains information about the dynamics of the diffusion process,

$$
C_{E}(\Omega) \equiv \frac{\left\langle E(\omega) E^{*}(\omega+\Omega)\right\rangle}{\langle|E(\omega)|\rangle\langle|E(\omega+\Omega)|\rangle}=\frac{\widetilde{J}_{z}\left(\sqrt{i \Omega / D+\alpha^{2}}, L\right)}{\widetilde{J}_{z}(\alpha, L)},
$$

where $E(\omega)$ is the complex field amplitude of the transmitted light for an incoming field of optical frequency $\omega$ and unit amplitude. The brackets \langle\rangle are used to explicitly denote ensemble averaging over all possible configurations of the disordered sample. We obtained the right hand side by assuming ergodicity and applying the Wiener-Khinchin theorem. 
The field-field correlation function in Eq. (12) is the exact result for diffuse transport through a slab using mixed boundary conditions and an exponential source distribution. Earlier results (Refs. [17,20]) are reproduced by using the simpler sheet source representation of Eq. (8) and choosing $z_{e 1}=z_{e 2}$ or $z_{e 1}=z_{e 2}=0$.

We will now turn to the intensity correlation function. This function relates two single channel transmission coefficients. The single channel transmission coefficient $T$ describes transmission from one input angle to one output angle. Integrating $T$ over all outgoing angles yields the total transmission coefficient $T_{\text {tot }}$. The intensity correlation function is defined as

$$
C_{I}(\Omega) \equiv \frac{\langle\delta T(\omega) \delta T(\omega+\Omega)\rangle}{\langle T(\omega)\rangle\langle T(\omega+\Omega)\rangle},
$$

where $\delta T(\omega) \equiv T(\omega)-\langle T(\omega)\rangle$. A well known approximation for the intensity correlation function is given by

$$
C_{I}(\Omega)=\left|C_{E}(\Omega)\right|^{2} .
$$

Equation (14) is referred to as the $C_{1}$ approximation and is valid for diffusive transport in multiply scattering media far away from the localization transition [21]. Equations (12) and (14) show that both $C_{E}(\Omega)$ and $C_{I}(\Omega)$ depend on the diffusion constant only by means of the reduced frequency $\Omega / D$. Fitting the frequency dependence of $C_{I}$ is a commonly used method to extract the diffusion constant from measured correlation functions.

It is instructive to introduce the characteristic traversal time for diffusive transmission $\tau_{t}$ [22], which is defined as the average time it takes a pulse of light to travel through the medium,

$$
\tau_{t} \equiv \frac{\int d t J_{z}(t, L) t}{\int d t J_{z}(t, L)}=i \lim _{\Omega \rightarrow 0} \frac{\partial C_{E}(\Omega)}{\partial \Omega} .
$$

The right hand side was obtained by rewriting the definition of $\tau_{t}$ in the Laplace domain representation and using Eq. (12). For zero absorption we find

$$
\tau_{t}=\frac{L_{e}^{2}-6 l^{2}-3 z_{e 1}^{2}-3 z_{e 2}^{2}}{6 D}+\frac{z_{e 1}^{3}+z_{e 2}^{3}}{3 L_{e} D}+O\left(e^{-L / l}\right) .
$$

The diffuse traversal time is of fundamental interest since it relates to the Thouless criterion for localization [23]. Furthermore, the time scale is of practical interest since measuring $\tau_{t}$ provides a method of determining the diffusion constant. Our result in Eq. (16) gives corrections of order $z_{e} L / D$ and higher to the value of $\tau_{t}=L^{2} / 6 D$ found by Landauer et al. [22]. These corrections are especially relevant when $L / z_{e}<10$, which is the case for thin samples or samples with a high extrapolation length due to internal reflection.

\section{B. Phase statistics}

The crucial difference between diffusion of particles and wave diffusion is interference. For this reason we are inter- ested in the phase of light that propagates through a scattering medium. An analysis of phase information is complementary to the analysis of the intensity and provides an independent method of measuring the traversal time $\tau_{t}$ and therefore the diffusion constant. We consider only single channel phase statistics, which means that we relate phase and amplitude for one input angle to the phase and amplitude for a single output angle.

Since the diffusion equation only describes the average intensity, an extension is needed in order to predict phase statistics. The statistical properties of the phase were predicted by van Tiggelen et al. [24] by assuming Gaussian statistics of the transmitted field [33]. This Gaussian assumption is valid when a high number of independent paths contributes to the field at the back surface of the random material. The central limit theorem predicts that in this situation the real and imaginary parts of the fields are described by a normal distribution [25]. Equivalently, the field amplitude is Rayleigh distributed and the phase $\phi$ has a uniform distribution between 0 and $2 \pi$. Neither the distribution of the intensity nor the distribution of the phase contains information about the diffusion process. Much more interesting is the probability distribution of the group velocity delay time $\phi^{\prime}$ $\equiv d \phi / d \omega$. This probability distribution reflects dynamic properties of the diffusion process and provides a method of measuring the diffusion constant. The statistics of the delay time $\phi^{\prime}$ were calculated in Ref. [24]. For this calculation the Gaussian field statistics were extended to describe the correlations of two fields at nearby frequencies. These correlations are given by the field-field correlation function. The resulting joint Gaussian distribution was subsequently used to calculate the probability distribution of the delay time,

$$
P\left(\widetilde{\phi}^{\prime}\right)=\frac{Q}{2\left[\left(\widetilde{\phi}^{\prime}-1\right)^{2}+Q\right]^{3 / 2}},
$$

where $\widetilde{\phi}^{\prime} \equiv \phi^{\prime} /\left\langle\phi^{\prime}\right\rangle$ and $Q$ is a dimensionless parameter. $\left\langle\phi^{\prime}\right\rangle$ and $Q$ can be calculated from the first and second terms in the Taylor expansion of the field-field correlation function: $C_{E}=1-i \tau_{t} \Omega-b \Omega^{2}+O\left(\Omega^{3}\right)$, which results in $\left\langle\phi^{\prime}\right\rangle=\tau_{t}$ and $Q \equiv 2 b / \tau_{t}^{2}-1[24]$.

In Ref. [24] the correlation function for a system with simplified boundary conditions was used to calculate $\tau_{t}$ and $Q$. Here it was shown that without absorption $Q$ equals $2 / 5$ while with absorption $Q$ is reduced. However, by carefully examining our solution for mixed boundary conditions, Eq. (12), we find that $Q$ increases above $2 / 5$ when the extrapolation lengths are nonzero.

The intensity-weighted delay time $W$ is a fundamental quantity since the sum of this quantity over all incoming and outgoing angles equals $\pi$ times the density of states in the medium $[24,26]$. The weighted delay time is defined as

$$
W \equiv T \phi^{\prime} .
$$

$T$ and $\phi^{\prime}$ are statistically dependent variables; for channels with a low transmission the probability distribution of $\phi^{\prime}$ is broader [27]. Because of the statistical dependency, the statistics of the weighted delay time cannot be deduced from the individual probability distributions of $T$ and $\phi^{\prime}$ and has 
to be calculated on its own. The probability distribution of $W$ was calculated in a similar way as the distribution of $\phi^{\prime}$ and is given by [24]:

$$
P(\tilde{W})=\frac{1}{\sqrt{1+Q}} \exp \left(\frac{-2|\tilde{W}|}{\operatorname{sgn}(\tilde{W})+\sqrt{1+Q}}\right),
$$

where sgn is the signum function [34] and $\tilde{W} \equiv W /\langle W\rangle$. The average weighted delay time was found [24] to relate to the diffuse traversal time according to $\langle W\rangle=\langle T\rangle \tau_{t}$.

The correlation function of the weighted delay time $C_{W}$ is defined as

$$
C_{W} \equiv \frac{\langle W(\omega) W(\omega+\Omega)\rangle}{\langle W(\omega)\rangle\langle W(\omega+\Omega)\rangle} .
$$

This correlation function was calculated in the $C_{1}$ approximation [Eq. (14)] using a joint Gaussian distribution that relates the fields at four frequencies [24]:

$$
C_{W}(\Omega)=\frac{1}{2 \tau_{t}^{2}}\left[\left|\frac{\partial C_{E}(\Omega)}{\partial \Omega}\right|^{2}-\operatorname{Re}\left(C_{E}(\Omega) \frac{\partial^{2} C_{E}^{*}(\Omega)}{\partial \Omega^{2}}\right)\right] .
$$

Microwave experiments showed that deviations from the $C_{1}$ approximation $\left(C_{2}\right.$ and $C_{3}$ correlations) cause $C_{W}$ to decay with frequency much slower than is described by Eq. (21) [27]. Therefore measuring $C_{W}$ provides a good way of testing the validity of the $C_{1}$ approximation and of looking for signs of localization.

\section{Diffusion in the time domain}

Although we found an exact solution to the diffusion equation in the frequency domain, the time-domain behavior is not obvious from Eq. (3). In this section we analyze diffusion in the time domain and we present an alternative technique for finding the diffusion constant. The time-resolved transmission can, in principle, be calculated by inverse Laplace transforming Eq. (7) by means of contour integration [28]. Unfortunately Eq. (7) has an infinite number of poles, none of which can be found analytically when the extrapolation lengths are nonzero. Using a different approach we will show that the diffusion constant can be found by analyzing only the long-time behavior of the transmitted flux.

A complete set of solutions to the diffusion equation [Eq. (1)] is given by

$$
I_{q_{z}, \theta}\left(\mathbf{q}_{\perp}, z ; t\right)=\sin \left(q_{z} z+\theta\right) \exp \left(-\left[q^{2}+\alpha^{2}\right] D t\right) \Theta(t),
$$

where $q^{2} \equiv q_{\perp}^{2}+q_{z}^{2}$ and $\Theta(t)$ is the Heaviside step function. In an infinite medium the longitudinal spatial frequency $q_{z}$ and phase $\theta$ can be chosen freely. In a finite slab, however, there is an infinite, discrete set of combinations of $q_{z}$ and $\theta$ for which the boundary conditions are fulfilled. For the boundary conditions given by Eqs. (2a) and (2b), permitted values of $q_{z}$ and the corresponding $\theta$ can be calculated numerically. Every solution for $q_{z}$ corresponds to two poles in Eq. (7) with $\eta= \pm i q_{z}$.
We will only calculate the long-time behavior of diffusion. In the long-time limit only the solution with the lowest $q_{z}$ survives, since, according to Eq. (22) all other solutions decay faster. We number this particular solution $q_{z 1}, \theta_{1}$. Now we are able to calculate the diffuse flux for $t \gg 1 / q_{z 1}^{2} D$,

$$
J_{z}\left(\mathbf{q}_{\perp}, z ; t\right)=-J_{0}\left(\mathbf{q}_{\perp}\right) \cos \left(q_{z 1} z+\theta_{1}\right) \exp \left(-\left[q_{z 1}^{2}+\alpha^{2}\right] D t\right),
$$

$J_{0}$ can be calculated by contour integrating Eq. (7) around the poles at $\eta= \pm i q_{z 1}$. In this article we are interested only in the exponential decay time of the transmitted flux and therefore will not explicitly specify $J_{0}$. For the total flux $\left(\mathbf{q}_{\perp}\right.$ $=0$ ) we find an exponential decay with a decay time $\tau_{d}$,

$$
\begin{aligned}
\tau_{d}^{-1} & =\left[q_{z 1}^{2}+\alpha^{2}\right] D, \\
& \approx\left[\frac{\pi^{2}}{L_{e}^{2}}+\alpha^{2}\right] D,
\end{aligned}
$$

where the approximate solution in Eq. (24b) was found by linearly extrapolating $I\left(\mathbf{q}_{\perp}, z ; t\right)$ at the slab boundaries (this is equivalent to the method of mirror images used in Ref. $[20,29])$ and $L_{e} \equiv L+z_{e 1}+z_{e 2}$ is an effective slab thickness. The approximate solution [Eq. (24b)] can be used for thick samples $\left(L \gg z_{e 1}, z_{e 2}\right)$.

It is interesting to notice the differences between the decay time $\tau_{d}$ and the diffuse traversal time $\tau_{t}$. The decay time $\tau_{d}$ describes the long-time decay rate of the energy density of diffuse light in the sample. This decay rate is given by the slowest term in Eq. (22) and does not depend on the distribution of the source intensity. The diffuse traversal time, on the other hand, has contributions from all terms in Eq. (22) and is mainly determined by the short-time transmission. The diffuse traversal time does depend on the distribution of the source intensity. Concluding, $\tau_{d}$ and $\tau_{t}$ are time scales that correspond to different aspects of diffusion. Therefore, the consistency of the diffusion model can be tested experimentally by measuring both $\tau_{d}$ and $\tau_{t}$ for a series of samples.

\section{Apparent nonexponential decay in a realistic experimental configuration}

In the previous section we found that the total transmitted flux decays single exponentially in the long-time limit. In an actual experimental geometry, however, it is not possible to collect all the transmitted light; only a finite area at the back surface of the sample can be imaged on the detector. We model the limited area by means of a Gaussian detection efficiency with a known waist $w_{d}$. Furthermore, we assume that the source light, $S\left(\mathbf{q}_{\perp}\right)$, has a Gaussian intensity distribution with waist $w_{s}$. The total intensity reaching the detector $J_{\text {det }}$ is found by integrating over all spatial frequencies $\mathbf{q}_{\perp}$,

$$
J_{\mathrm{det}}(t)=\frac{\pi w_{d}^{2}}{2} \int d \mathbf{q}_{\perp} J_{z}\left(\mathbf{q}_{\perp} ; L, t\right) \exp \left(-\frac{1}{8} q_{\perp}^{2} w_{d}^{2}\right),
$$

The intensity profile at the sample surface is time dependent according to Eq. (22) since modulations with a high spatial frequency $q_{\perp}$ decay faster than those with a low spatial fre- 
quency. Since we detect only the flux from a finite area, our detection efficiency is time dependent as well. We define $\tau_{f}$ $\equiv\left(w_{s}^{2}+w_{d}^{2}\right) / 8 D$, being the characteristic time scale for the time-dependent detection efficiency, and find the total detected flux from Eq. (25),

$$
J_{\text {det }}(t)=\frac{w_{d}^{2} /(8 D)}{t+\tau_{f}} J_{z}\left(\mathbf{q}_{\perp}=0 ; L, t\right) .
$$

This equation shows that a finite detection area imposes a nonexponential envelope on the detected transmission and increases the detected decay rate. For thicker samples the additional decay will be more pronounced since the diffuse decay, as described by $\tau_{d}$, is slower. As a result, the diffusion constant found from a linear fit of $\ln J(t)$ is structurally overestimated. Usually the prefactor in Eq. (26) is omitted, corresponding to the assumption that the detection system collects light from a large area $\left(w_{d} \gg w_{s}\right)$. The consequences of omitting this correction can, however, be significant: in our experimental configuration the correction results in up to a $25 \%$ modification of the measured diffusion constant.

Naturally, the finite-area correction given by Eq. (26) equally applies for diffusion in the frequency domain. Unfortunately, it is inconvenient to apply the correction in the frequency domain analytically. Therefore we use a numerical fast Fourier transform to correct the frequency-resolved transmission, Eq. (7), and all derived quantities.

\section{E. Five ways of measuring the diffusion constant}

In Secs. II A-II C we presented methods to calculate the frequency correlations, the phase statistics and the transient behavior of light diffusing through a slab of randomly scattering material. Two important time scales were identified: the diffuse traversal time $\tau_{t}$ and the exponential decay time $\tau_{d}$.

In our experiments we will test the consistency of the diffusion model quantitatively by extracting the diffusion constant from experimental data using five different techniques. If the model is valid, we expect all techniques to yield the same diffusion constant when the boundaries and the source intensity distribution are accounted for correctly. Furthermore, the methods will only give the same results when coherent transmission, higher order correlations and Anderson localization do not play a role. Therefore a comparison of the diffusion constants, measured using different methods, provides an excellent way of testing our diffusion model.

Method I: In the first method, the diffusion constant is found from the diffuse traversal time $\tau_{t}$. The diffuse traversal time is obtained from time-resolved transmission using the definition in Eq. (15). After applying the finite-area correction, the diffusion constant is found by means of Eq. (16). Since the transmitted intensity decays exponentially the value of $\tau_{t}$ depends mainly on the transmission at short time scales.

Method II: The second method is to measure the decay time $\tau_{d}$ by fitting the long-time decay of the transmitted flux. Subsequently, Eq. (24a) is used to find the diffusion constant. Since Method II relies on the time-resolved transmission at long time scales, the parts of the data used in Method I and Method II are nearly independent.

Method III: In the third method, the intensity correlation function is extracted from frequency-resolved measurements. Fitting Eq. (14) to the measured correlation function yields the diffusion constant.

Method IV: The fourth method relies on the measured optical phase and makes use of the statistics derived for the phase of diffuse light. When the field obeys Gaussian statistics $\left\langle\phi^{\prime}\right\rangle$ equals the diffuse traversal time $\tau_{t}$. Consequently, Eq. (16) can be used to extract the diffusion constant from the measured phase. Since Method IV only uses phase information and Methods III only uses the measured intensity, these two methods are fully independent. In the case of Anderson localization, the transmitted field does not obey Gaussian statistics. The diffuse traversal time from Methods $\mathrm{I}$ and $\mathrm{V}$ is influenced by resonant tunneling through localized modes and will be significantly higher than the average phase delay time measured using Method IV [11].

Method V: In the last method the diffusion constant is extracted from measurements of the weighted delay time. With $\langle W\rangle /\langle T\rangle=\tau_{t}$ we find the diffuse traversal time. As in Methods I and IV we calculate the diffusion constant using Eq. (16). It has been shown [30] that Method V is mathematically equivalent to Method I. Therefore, we will only use Method V to verify the consistency of our data processing.

All together we now have five different methods of measuring the diffusion constant. A comparison of the results of these methods provides a thorough test of the diffusion model and the phase statistics. Furthermore it enables an unambiguous determination of the diffusion constant.

\section{EXPERIMENT}

We have presented a theoretical framework connecting time-resolved measurements to phase statistics and frequency correlations. In order to test this framework, we need to measure both the amplitude and the phase of the multiple scattered light over a range of optical frequencies simultaneously. We perform these measurements using the technique of femtosecond pulse interferometry as described in Ref. [12]. This technique involves an incoherent light source and two interferometers. As is explained below, the coherence time of the light source should be smaller than the relative delay that can be achieved in the interferometers. In our case the light source is a mode-locked Ti:sapphire laser (Tsunami, Spectra Physics) operating at $775 \mathrm{~nm}$ producing femtosecond pulses with a bandwidth of about $6 \mathrm{~nm}$. The technique, however, is not limited to pulsed lasers. Any light source with sufficient bandwidth, such as a super luminescent diode or even a conventional lamp, can be used. In our experiment we collect light from only a single speckle spot. Therefore we require a high source intensity (about $50 \mathrm{~mW}$ at the sample surface). Furthermore, we need to focus the beam in order to have large speckle spots. The excellent beam quality and high power favor the use of a mode-locked laser.

The first of the two interferometer is of the Mach-Zehnder type and is shown schematically in Fig. 1. A beamsplitter 


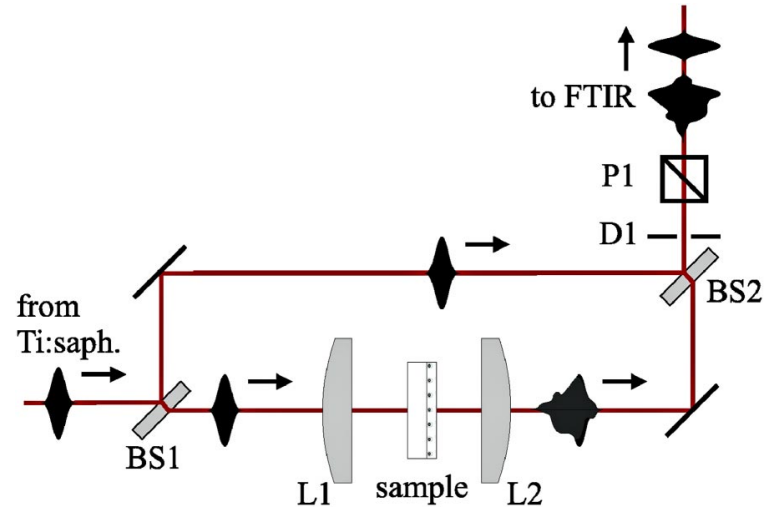

FIG. 1. Schematic representation of the Mach-Zehnder interferometer used in the setup. The first beamsplitter (BS1) divides the light between a reference arm and a signal arm. The light in the signal arm is focused on the sample by lens L1. The transmitted speckle pattern is collimated by lens L2 and recombined with the reference beam at beamsplitter BS2. Since the reference arm is a few millimeters shorter than the sample arm, the signal pulse does not overlap the reference pulse temporally. Finally, aperture $D 1$ selects an area that is smaller than the typical speckle size and polarizer P1 blocks light with a polarization perpendicular to that of the reference beam in order to increase the signal to noise ratio. The beam containing the signal pulse and the reference pulse is propagated into a scanning interferometer (FTIR). When a sample is placed in the signal arm, only a fraction of the incident light reaches the FTIR. In order to balance the interferometer, we use beamsplitters (BS1 and BS2) that reflect approximately $4 \%$.

divides the incoming light between a signal arm and a reference arm. In the signal arm the light of the laser is focused on the sample to a waist diameter of approximately $30 \mu \mathrm{m}$ using a lens with a focal length of $6 \mathrm{~cm}$. In order to probe different random configurations of scatterers we illuminate different areas of the sample by translating the sample perpendicular to the incoming beam. For every sample position the transmitted light forms a different volume speckle pattern. The speckle is collimated using a second $6 \mathrm{~cm}$ lens and an area smaller than a typical speckle spot is selected from the pattern using an aperture with a diameter of $0.8 \mathrm{~mm}$. At the second beamsplitter the light transmitted through this aperture is combined with the reference pulse yielding a beam with two temporally separated pulses.

The double-pulsed signal is directed into a Fourier transform infrared interferometer (FTIR). The FTIR (Biorad FTS$60 \mathrm{~A})$ is a Michelson interferometer and scans the delay time between two copies of the signal. A detector directly behind the FTIR obtains the field autocorrelation function of the pulse pair as a function of the extra pathlength in the scanning arm of the interferometer. It takes about $40 \mathrm{~s}$ to measure the autocorrelation function. During this time, the speckle pattern has to be stationary and the interferometer stable with subwavelength accuracy. For this reason only solid samples can be used.

Because of the temporal separation of the signal and reference pulses, it is possible to isolate the cross correlate $C(t)$ of the signal pulse with the reference pulse [35]. In the frequency domain the cross correlate is given by

$$
C(\omega)=|S(\omega)|^{2} H_{s}(\omega) H_{r}(\omega) E(\omega),
$$

where $S(\omega)$ is the spectrum of the incoming pulse, $H_{s}(\omega)$ and $H_{r}(\omega)$ are the transfer functions of the signal and reference arm of the Mach-Zehnder interferometer, respectively and $E(\omega)$ is the transfer function of the sample that we wish to extract. In order to find the transfer function of the sample, the cross correlate is measured with and without the sample consecutively. Dividing the two functions yields the complex transfer function $E(\omega)$ containing both the phase and the amplitude of the transmitted light. Now the time-resolved field transmission $E(t)$ can in principle be obtained by means of an inverse Fourier transform. In practice, however, the bandwidth of the transfer function is limited by the bandwidth of the source pulse. Outside this bandwidth the measured transfer function is dominated by noise, therefore additional filtering is required before calculating $E(t)$. We use a Chebyshev filter for filtering in order to have a minimum effect of side lobes and a maximum time resolution.

In the experiment the signal and the reference beams have to overlap both at the aperture and at the detector in order to cause an interference signal. This condition implies that both the direction and the position of the signal beam are fixed and, as a result, the detection is limited to light emitted from a small area of the sample surface. Based on the geometry of the setup we approximate the detection area by a Gaussian curve with a waist of $w_{d}=10 \mu \mathrm{m}$.

We perform the measurements on samples consisting of a layer of rutile $\mathrm{TiO}_{2}$ particles with a diameter between $150 \mathrm{~nm}$ and $290 \mathrm{~nm}$ that are deposited on a substrate of fused silica. The titania grains have a refractive index of approximately 2.8. The extrapolation lengths can be calculated from the effective refractive index $n_{\text {eff }}$ of the medium [17]. The effective index can adequately be estimated from Mie theory [31]. For our samples we find $n_{\mathrm{eff}}=1.34$ and the corresponding extrapolation lengths are $z_{e 1} / l=0.69$ for the left boundary and $z_{e 2} / l=1.71$ for the right boundary. We measured the total transmission as a function of sample thickness and found a transport mean free path of $l$ $=0.97 \pm 0.10 \mu \mathrm{m}$ by fitting Eq. (11) to the data.

Our samples range in thickness between $1.5 \pm 0.3 \mu \mathrm{m}$ and $18.0 \pm 0.3 \mu \mathrm{m}$. Since the samples are on a substrate that is much thicker than the layer of titania, it is necessary to compensate for the extra delay in the substrate. In order to accurately determine the extra pathlength, we direct the light that is reflected from the substrate into the FTIR without repositioning the sample. The thickness of the substrate is deduced from the time delay between the reflections from the front and the back of the substrate.

With the setup described in this section we are able to measure the complex transfer function of random media. Below we analyze these transfer functions in the time domain, the frequency domain and by looking at the phase statistics.

\section{RESULTS}

\section{A. Time domain measurements}

First we consider the decay in time of a transmitted pulse. The inset in Fig. 2 shows the raw data obtained by measuring 


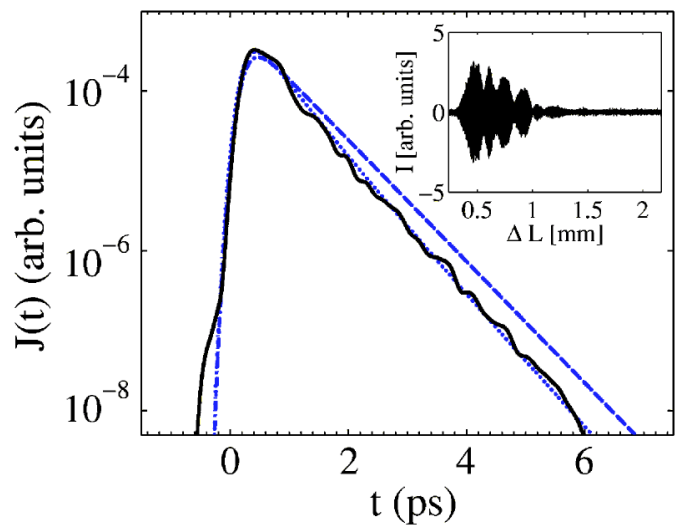

FIG. 2. Time-resolved intensity transmission for a $10.1 \mu \mathrm{m}$ thick sample consisting of $\mathrm{TiO}_{2}$ grains. The observed nonexponential decay (solid line) agrees with the finite-area correction, Eq. (26), over four decades. Theoretical curves are obtained from Eq. (7) and the shape of the filter. The dotted line was corrected for the time-dependent detection efficiency due to focusing, using Eq. (26) with $w_{s}=15 \mu \mathrm{m}$ and $w_{d}=10 \mu \mathrm{m}$ as estimated from the experimental configuration and a fitted diffusion constant of $D=27.0 \mathrm{~m}^{2} \mathrm{~s}^{-1}$. The dashed line is the uncorrected curve for the same diffusion constant. The inset shows an example of the interference signal at the detector as a function of the delay length in the scanning interferometer. The average intensity transmission is obtained from 50 such measurements performed on different areas of the sample.

the cross correlate at a single position of the sample. For different positions of the sample the transmitted pulse is distorted differently. We extract $E(t)$ for every measurement as described in Sec. III and average the corresponding intensities over 50 sample positions to obtain the normalized transmission $J(t) \equiv\left\langle|E(t)|^{2}\right\rangle$. Figure 2 shows the time-resolved intensity transmission $J(t)$ for a $10.1 \mu \mathrm{m}$ thick sample. We find that in the long-time limit $J(t)$ has a nearly exponential decay for more than four decades. The measurements are fitted with the theoretical curve obtained from Eq. (7) convolved with the frequency filter that was used in the processing of the raw data. We find a good fit for a diffusion constant of $D$ $=27.0 \mathrm{~m}^{2} \mathrm{~s}^{-1}$, taking into account the effect of the limited area of detection. For comparison, the theoretical curve without correction for the detected area is also shown in Fig. 2. The corrected curve exhibits a significantly faster decay, especially for $t<2$ ps.

In order to analyze the decay of $J(t)$ more quantitatively, we extract the diffuse traversal time $\tau_{t}$ and the decay time $\tau_{d}$ from the measured flux. In Sec. IV D the diffusion constant will be calculated from these two times scales using Methods I and II (Sec. II E) respectively. The first time scale $\tau_{t}$ is obtained from the time-resolved transmission directly using Eq. (15). The second time scale $\tau_{d}$ is extracted from an exponential fit of the intensity decay. We fit the data between $t>\tau_{d}$ and the point where the intensity is dropped below the noise. It was found that the decay in the first half of this range is always significatly faster than decay in the second half, as is predicted by Eq. (26). In Fig. 3 the decay time $\tau_{d}$ is compared to the diffuse traversal time $\tau_{t}$. It was shown in Sec. II that the differences between the two time scales are caused by surface effects and the limited detection area. We

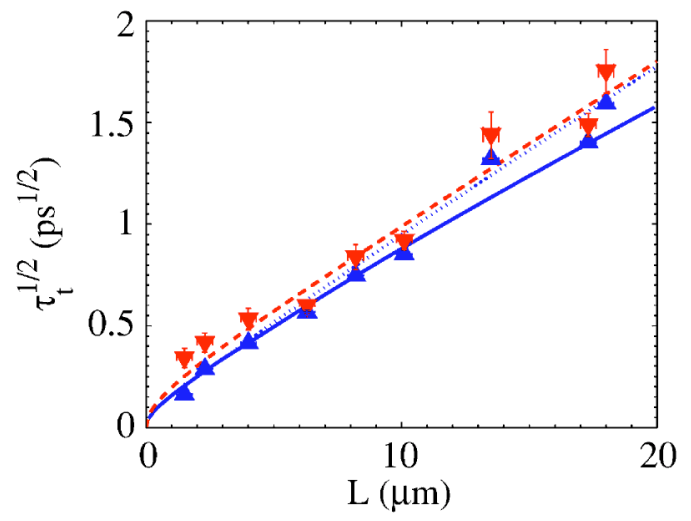

FIG. 3. Diffuse traversal time $\tau_{t}$ (triangles pointing upward), and scaled decay time $\tau_{d} \pi^{2} / 6$ (triangles pointing downward), as a function of sample thickness. $\tau_{d}$ is found by fitting the exponential decay of $J(t)$ (Method II); the error bars indicate the values found from fitting the first part of the decay (lowest value) and the last part of the decay (highest value). The dashed line is the theoretical value of $\tau_{d}$. The diffuse traversal time is found from the timeresolved intensity transmission obtained by numerically evaluating Eq. (15) (Method I). Without taking into account detection efficiency, theory (dotted line) predicts that the two time scales converge for thick samples. When the theory is compensated for the effect of a finite detection area (solid line), this convergence is lost, in agreement with the experimental data.

find a good agreement with theory for the decay time as well as for the traversal time, both using the same fitted average diffusion constant of $D=25.5 \mathrm{~m}^{2} \mathrm{~s}^{-1}$.

\section{B. Phase statistics}

An independent way of measuring the diffuse traversal time is by analyzing the phase information. For different positions of the sample we obtain the phase $\phi$ from the complex transfer function $E(\omega)$ that was measured using the technique described in Sec. III. For all different frequencies in the $6 \mathrm{~nm}$ bandwidth of the measurements, we calculate the delay time $\phi^{\prime}(\omega) \equiv d \phi(\omega) / d \omega$ and the weighted delay time $W(\omega) \equiv \phi^{\prime}(\omega)|E(\omega)|^{2}$. By binning the values of $\phi^{\prime}$ and $W$, we obtain the probability distributions shown in Fig. 4. The distributions are in good agreement with the predicted functional forms from theory, Eq. (17) and Eq. (19). This agreement is a clear experimental proof that the transmitted light is described well by a circular complex Gaussian distribution. For a sample with a thickness of $10.1 \mu \mathrm{m}$, the characteristic parameter $Q$ determining the width of the distribution is calculated to be $Q=0.44$. The experimental data gives $Q=0.47$, corresponding to a slightly lower maximum of $P\left(\phi^{\prime}\right)$. The high value of $Q$ indicates that there is no measurable effect of absorption (which would decrease $Q$ ). Moreover, $Q$ is clearly larger than the value of $2 / 5$ predicted in Ref. [24]. This observation shows that even for thick samples $(L \approx 10 l)$ the effect of reflections at the surfaces cannot be neglected.

We obtain the diffuse traversal time using $\tau_{t}=\left\langle\phi^{\prime}\right\rangle$ (Method IV) and $\tau_{t}=\langle W\rangle$ (Method V) and compare these results to the value found from the time-resolved intensity 

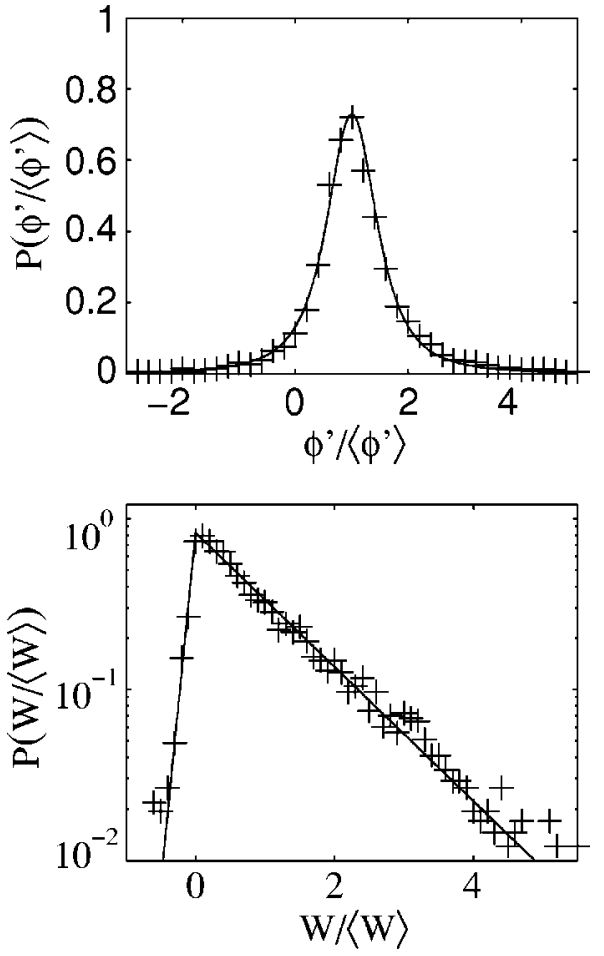

FIG. 4. Probability distributions for the delay time $\phi^{\prime}$ (top) and the weighted delay time $W$ (bottom) as measured in a $10.1 \mu \mathrm{m}$ thick $\mathrm{TiO}_{2}$ sample. The dimensionless parameter $Q$ characterizes the width of these distributions. We find $Q=0.47$ from a fit of the theoretical curves given by Eqs. (17) and (19) (solid lines). The average values of $\phi^{\prime}$ and $W$ are used in Methods IV and V, respectively, to find the diffusion constant.

measurements (Method I). Figure 5 shows $\tau_{t}$, as obtained by these three different methods. The values of $\left\langle\phi^{\prime}\right\rangle$ coincide almost perfectly with $\tau_{t}$ found from time-resolved analysis. This agreement again confirms the excellent validity of the $C_{1}$ approximation and the theory of phase statistics.

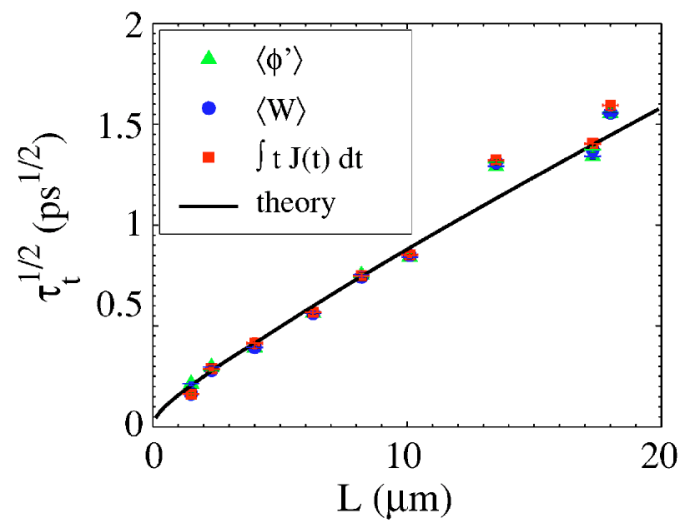

FIG. 5. Diffuse traversal time $\tau_{t}$ measured using three different techniques. Method I (squares) calculates $\tau_{t}$ from the time-resolved intensity. Method IV (triangles) obtains $\tau_{t}$ from the measured optical phase alone, whereas Method V (circles) uses the intensityweighted phase information. The excellent agreement indicates that the transmitted field is described by Gaussian distribution. The solid line are the theoretical values for a diffusion constant of $D$ $=25.5 \mathrm{~m}^{2} \mathrm{~s}^{-1}$.

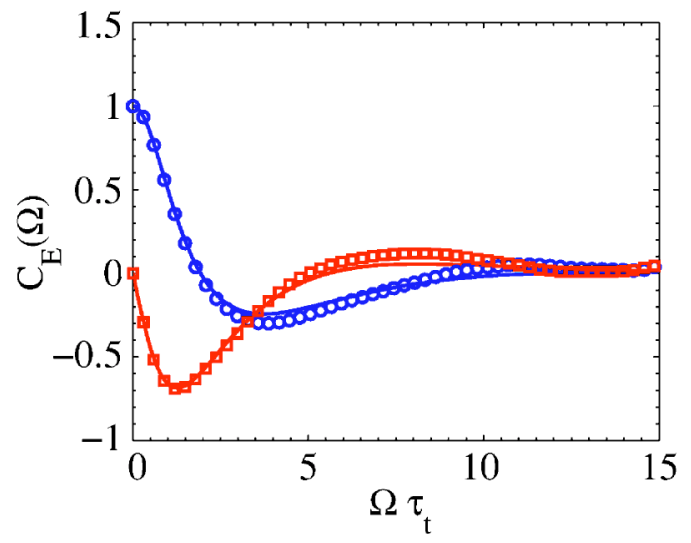

FIG. 6. Measured field correlation function $C_{E}$ (real part: circles, imaginary part: squares) for a $\mathrm{TiO}_{2}$ sample of thickness $10.1 \mu \mathrm{m}$ as a function of the frequency difference $\Omega$ between two optical frequencies. The horizontal axis is scaled by $\tau_{t}^{-1}$ as found by measuring $\left\langle\phi^{\prime}\right\rangle$. Excellent agreement with theory (solid lines) confirms the diffusion model with boundary corrections.

As expected, the results from Methods I and V agree very well. Although these methods are equivalent in theory, the time-domain data, on which Method I is based, have been filtered (see Sec. III), whereas Method V uses the unfiltered frequency-domain measurements directly. Since the differences between the values obtained by Methods I and V are minute, we conclude that the determination of $\tau_{t}$ is insensitive to frequency domain filtering. In Sec. IV D we will use $\tau_{t}$ to extract the diffusion constant for each sample.

\section{Frequency domain measurements}

In Secs. IV A and IV B we presented measurements of the traversal time $\tau_{t}$ and $Q$, the characteristic parameter for phase statistics. These two parameters are related to the first and second order terms in the Taylor expansion of the field-field correlation function $C_{E}$ around $\Omega=0$. In this section we go a step further and investigate the full frequency correlation functions of the transmitted light. We investigate the fieldfield correlation function, the intensity correlation function and the correlation function of the weighted delay time consecutively.

We first look at the field-field correlation function. This function is related to the time-resolved intensity transmission by a Fourier transform and provides an alternative way of studying the propagation of diffuse intensity without having to worry about possible artefacts introduced by filtering. In analyzing the time-resolved transmission plotted in Fig. 2 we only extracted two parameters, $\tau_{t}$ and $\tau_{d}$. Whereas the measured time-resolved transmission curve showed some minor fluctuations compared to theory, the field-field correlation function is perfectly smooth up to $\Omega=15 \tau_{t}$ and we find that the theoretical curve matches the experimental data very well, as is shown in Fig. 6.

In order to test the $C_{1}$ approximation [Eq. (14)] directly, we examine the intensity correlation function $C_{I}(\Omega)$. The presence of long range $\left(C_{2}\right)$ and infinite range $\left(C_{3}\right)$ correlations would show up by comparing the intensity correlation 


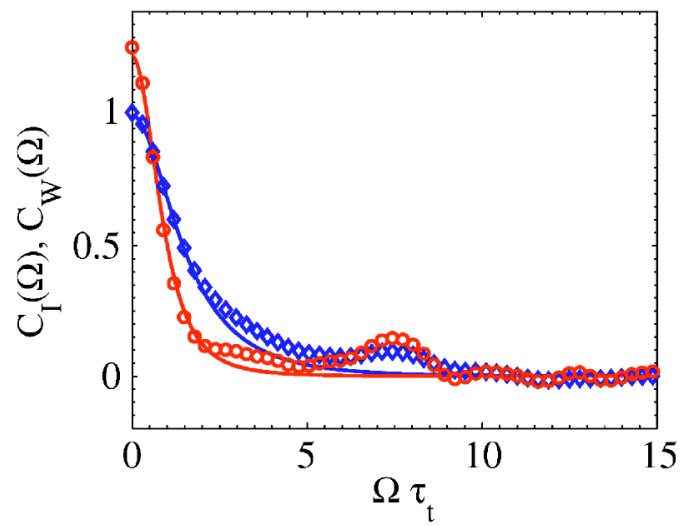

FIG. 7. Measured correlation functions for the intensity $C_{I}(\Omega)$ (diamonds), and the weighted delay time $C_{W}(\Omega)$ (circles), in a $10.1 \mu \mathrm{m}$ thick $\mathrm{TiO}_{2}$ sample. The solid lines are the $C_{1}$ approximations for both correlation functions. Except for some spurious oscillations, agreement with theory is evident.

function to the $C_{1}$ contribution. In our experiment, however, we find a good agreement to the $C_{1}$ theory as is shown in Fig. 7. At $\Omega \tau_{t} \approx 8$ a slight deviation of unknown origin is found in the correlation function. Surprisingly this deviation was absent in the field-field correlation function $C_{E}$. We determine the diffusion constant by fitting the intensity correlation function and find a diffusion constant of $27 \pm 3 \mathrm{~m}^{2} \mathrm{~s}^{-1}$ for a $10.1 \mu \mathrm{m}$ thick sample.

Finally, we present the correlation function for the weighted delay time $C_{W}(\Omega)$ in Fig. 7. Apart from the same deviations that were found in the intensity correlation function, the agreement with Eq. (21) is evident. This observation provides the first experimental confirmation of the correlation function of the weighted delay time at optical frequencies.

\section{The diffusion constant}

Altogether we have presented five different methods of determining the diffusion constant experimentally. Methods I and II use the measured time-resolved intensity transmission to find two time scales, $\tau_{d}$ and $\tau_{t}$, from which the diffusion constant can be calculated. Subsequently, we showed that $\tau_{t}$ can be obtained from phase statistics in two different ways by analyzing the delay time (Method IV) and the weighted delay time (Method V). Finally, we measured the diffusion constant by fitting the intensity correlation function (Method III). The results of these five methods are summarized in Fig. 8 for nine samples of different thickness. We find that all different methods yield the same diffusion constant, within the experimental accuracy, for a given sample. This observation is an experimental proof of the consistency of the diffusion model that was presented in Sec. II. An average diffusion constant of $D=25.5 \pm 1.0 \mathrm{~m}^{2} \mathrm{~s}^{-1}$ is found; individual samples with different thickness have slightly different values of the diffusion constant ranging from $19 \mathrm{~m}^{2} \mathrm{~s}^{-1}$ to $28 \mathrm{~m}^{2} \mathrm{~s}^{-1}$. Since these variations in the diffusion constants are reproduced for all methods, we conclude that the scatter is a result of the varying sample structure and that it is not the result of a measurement error.
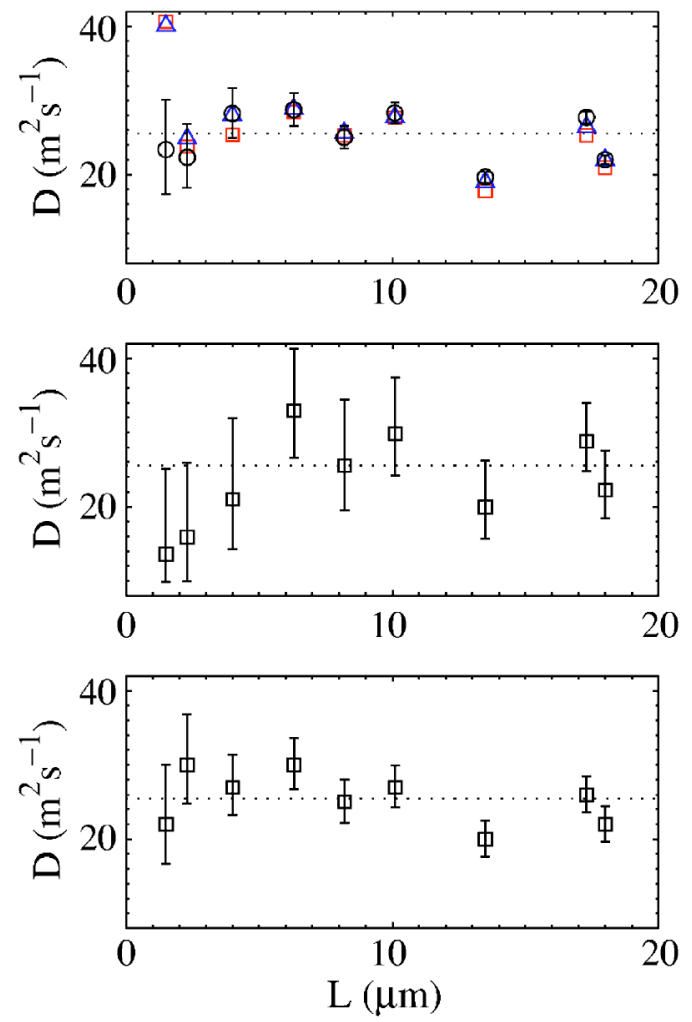

FIG. 8. Measured diffusion constant for samples of different thicknesses obtained in five different ways. The top figure shows the diffusion constant obtained from the diffuse traversal time $\tau_{t}$. We measured the traversal time directly in the time domain (Method I, squares), by extracting the average of the delay time $\left\langle\phi^{\prime}\right\rangle$ (Method IV, circles), and the weighted delay time $\langle W\rangle$ (Method V, triangles). Error bars are only presented for $\left\langle\phi^{\prime}\right\rangle$, but the errors in the two other values are comparable. The diffusion constants in the middle figure are calculated from the decay time $\tau_{d}$ which is obtained by fitting the decay of the transmitted flux (Method II). The bottom plot displays the diffusion constants obtained from fitting the intensity correlation function (Method III). Except for the two thinnest samples, the consistency of the five different measurement methods is evident.

The error bars in Fig. 8 are derived from the uncertainty in the sample thickness and the uncertainty in determining the actual value $\left(\tau_{t}, \tau_{d}\right.$ or the curve fit to $\left.C_{I}\right)$ that was used to calculate the diffusion constant. For thinner samples we find larger error bars since the uncertainty in the thickness is relatively large. For the three methods that are based on the traversal time $\tau_{t}$ we find that the uncertainty in $D$ decreases with increasing sample thickness. The determination of the decay time $\tau_{d}$, on the other hand, becomes increasingly more inaccurate because of the nonexponential decay of the transmitted intensity.

The diffusion constants obtained by fitting the decay of the transmitted intensity (Method II) appear to be slightly lower for thinner samples. This behavior is consistent with earlier observations [32]. It should be noted, however, that the decay time in these samples is comparable to the timedomain resolution. Measurements based on phase information (which are not limited by the time resolution) show no thickness dependence of the diffusion constant. 
For the thinnest sample we find different diffusion constants depending on which method we use. The difference is most apparent when comparing Methods I and V to Method IV. Both the direct time-domain measurement of $\tau_{t}$ (Method I) and the average weighted delay time (Method V) are lower than expected, resulting in significantly higher values of the measured diffusion constant. The difference in observed diffusion constants is a clear indication that the transmitted field does not have a Gaussian distribution. The discrepancy between the different values of $\tau_{t}$ is consistent with an increased transmission at short times. Therefore this observation suggests an influence of coherent transmission or single scattering. Especially, it shows that shorter traversal times are associated with higher intensities, since the low values of $\tau_{t}$ are not reproduced in the unweighted delay time measurements (Method IV).

\section{CONCLUSION}

Pulse interferometric measurements allow a sensitive determination of the complex transfer function of a random medium. This transfer function can be used to calculate the time-resolved intensity, the frequency-resolved intensity and notably the phase of diffuse light. These three complementary sets of data are obtained in a single measurement.

In Sec. II we presented a consistent theoretical framework to interpret the experimental data. The framework is built around an exact solution to the diffusion equation with mixed boundary conditions. Our solution is a generalized version of the result presented in Ref. [17] and uses a more accurate description of the source intensity distribution. A new result is the nonexponential envelope that is imposed on the detected flux due to a finite detection area. This envelope contributes to the detected intensity decay and should be taken into account when extracting the diffusion constant.

By analyzing the diffusion theory both in the time domain and in the frequency domain we have identified two relevant time scales. The first is the diffuse traversal time $\tau_{t}$. The traversal time is the natural time scale in the analysis of phase statistics and correlation functions. The second time scale is the decay time $\tau_{d}$ associated with the exponential decay of diffuse intensity. These two times are affected differently by the boundary conditions and the finite area of detection.

We measured the diffusion constant using five different methods, which allowed a comparison of time domain measurements, frequency domain measurements and phase measurements. We showed that the most accurate results are obtained by calculating the diffusion constant from the traversal time. The traversal time is obtained directly from intensity measurements (Method I) or from phase measurements (Methods IV and V). These three methods have in common that they provide a direct, unambiguous way of associating a time scale to the transmission of light. This makes them equally suited for the regimes of ballistic transmission, diffusion and localization. In fact, a comparison of the values obtained using Methods IV and V can distinguish between these regimes [11]. Method I has the additional advantage that it does not require phase information. Therefore this method is still applicable in situations where pulsed interferometry cannot be used to measure the phase, for example, in the analysis of colloidal suspensions that are nonstationary samples. Methods II and III use a curve fit of, respectively, the time-resolved intensity and the frequency correlation function of the intensity. Such techniques that rely on fitting of the experimental data are less accurate than direct calculation of the diffusion constant and explicitly use the diffusion model. Therefore these two methods are the least attractive of the five methods.

For our samples consisting of $\mathrm{TiO}_{2}$ particles we found that the results of the five complementary techniques agree almost perfectly for samples thicker than twice the mean free path. Our observations are strong experimental proof of the validity of the diffusion model and the phase statistics theory.

Finally we measured the correlation function of the weighted delay time. To our knowledge, this is the first report of such a measurement at optical wavelengths. The experimental data agree very well with the measured intensity correlation function and the predictions from phase statistics theory. These measurements demonstrate that it is possible to record phase-related correlation functions at optical wavelengths.

Our analysis clearly shows that care has to be taken in including proper boundary conditions and correcting for the detection efficiency, even for samples much thicker than the mean free path. Provided these effects are taken into account properly, the model used to describe the propagation of light through a random medium is consistent for all different methods of analysis. Our experiment demonstrates the versatility and reliability of pulse-interferometric measurements and validates the use of phase-sensitive quantities for the identification of long range correlations and possibly the localization of light.

\section{ACKNOWLEDGMENTS}

We thank Boris Bret and Allard Mosk for stimulating discussions. This work is part of the research program of the "Stichting voor Fundamenteel Onderzoek der Materie (FOM)," which is financially supported by the "Nederlandse Organisatie voor Wetenschappelijk Onderzoek (NWO)."
[1] D. J. Pine, D. A. Weitz, P. M. Chaikin, and E. Herbolzheimer, Phys. Rev. Lett. 60, 1134 (1988).

[2] J. F. de Boer and T. E. Milner, J. Biomed. Opt. 7, 359 (2002).

[3] F. Scheffold and G. Maret, Phys. Rev. Lett. 81, 5800 (1998).
[4] M. P. van Albada and A. Lagendijk, Phys. Rev. Lett. 55, 2692 (1985).

[5] P. E. Wolf and G. Maret, Phys. Rev. Lett. 55, 2696 (1985).

[6] N. F. Mott, Metal-Insulator Transitions (Taylor \& Francis, 
London, 1974).

[7] P. W. Anderson, Philos. Mag. B 52, 505 (1985).

[8] A. A. Chabanov, M. Stoytchev, and A. Z. Genack, Nature (London) 404, 6780 (2000).

[9] D. S. Wiersma, P. Bartolini, A. Lagendijk, and R. Righini, Nature (London) 390, 671 (1997)

[10] F. Scheffold, R. Lenke, R. Tweer, and G. Maret, Nature (London) 398, 206 (1999).

[11] A. A. Chabanov and A. Z. Genack, Phys. Rev. Lett. 87, 233903 (2001).

[12] R. H. J. Kop and R. Sprik, Rev. Sci. Instrum. 66, 5459 (1995).

[13] P. M. Johnson, A. Imhof, B. P. J. Bret, J. G. Rivas, and A. Lagendijk, Phys. Rev. E 68, 016604 (2003).

[14] E. Akkermans, P. E. Wolf, and R. Maynard, Phys. Rev. Lett. 56, 1471 (1986).

[15] M. C. W. van Rossum and T. M. Nieuwenhuizen, Rev. Mod. Phys. 71, 313 (1999).

[16] A. Lagendijk, R. Vreeker, and P. de Vries, Phys. Lett. A 136, 81 (1989)

[17] J. X. Zhu, D. J. Pine, and D. A. Weitz, Phys. Rev. A 44, 3948 (1991).

[18] H. S. Carslaw and J. C. Jaeger, Conduction of Heat in Solids (University Press, Oxford, 1959), 2nd ed.

[19] J. G. Rivas, R. Sprik, C. M. Soukoulis, K. Busch, and A. Lagendijk, Europhys. Lett. 48, 22 (1999).

[20] A. Z. Genack and J. M. Drake, Europhys. Lett. 11, 331 (1990).

[21] R. Berkovits and S. Feng, Phys. Rep. 238, 135 (1994).

[22] R. Landauer and M. Büttiker, Phys. Rev. B 36, 6255 (1987).
[23] D. J. Thouless, Phys. Rev. Lett. 39, 1167 (1977).

[24] B. A. van Tiggelen, P. Sebbah, M. Stoytchev, and A. Z. Genack, Phys. Rev. E 59, 7166 (1999).

[25] J. W. Goodman, Statistical Optics (Wiley, New York, 2000).

[26] G. Iannaccone, Phys. Rev. B 51, 4727 (1995).

[27] A. Z. Genack, P. Sebbah, M. Stoytchev, and B. A. van Tiggelen, Phys. Rev. Lett. 82, 715 (1999).

[28] J. W. Brown and R. V. Churchill, Complex Variables and Applications (McGraw-Hill, New York, 1996), 6th ed.

[29] M. B. van der Mark, Propagation of Light in Disordered Media: A Search For Anderson Localization (University of Amsterdam, Amsterdam, 1990).

[30] P. Sebbah, O. Legrand, and A. Z. Genack, Phys. Rev. E 59, 2406 (1999).

[31] P. N. den Outer and A. Lagendijk, Opt. Commun. 103, 169 (1993).

[32] R. H. J. Kop, P. de Vries, R. Sprik, and A. Lagendijk, Phys. Rev. Lett. 79, 4369 (1997).

[33] Reference [24] also gives corrections for non-Gaussian field statistics. We do not consider these in this paper.

[34] We corrected the original formula in Ref. [24] that contains a Heaviside step function in place of the signum function.

[35] Strictly speaking, temporal separation of the two pulses is not a necessity. As long as the pathlength difference is much larger than the correlation lengths of the light in the signal and the reference arms, the cross correlate can be isolated. Therefore incoherent continuous sources can be used instead of the pulsed laser. 